\title{
Preseptal cellulitis as a rare presentation of the oculocutaneous anthrax
}

\author{
Fatih Cem Gül ${ }^{1}$, Burak Turgut ${ }^{2 *}$, Mehmet Kaan Kaya ${ }^{3}$ and Ülkü Çeliker ${ }^{2}$ \\ ${ }^{1}$ Elazığ Education and Research Hospital, Elazig, Turkey \\ ${ }^{2}$ Firat University, School of Medicine, Department of Ophthalmology, Elâzig, Turkey \\ ${ }^{3}$ Avrupa Göz Hastanesi, Elazig, Turkey
}

\begin{abstract}
We aimed to report a case of oculocutaneous anthrax misdiagnosed as preseptal cellulitis at the presentation. A 14-year-old male patient was referred to emergency service in our university hospital with preliminary diagnosis of angioedema. He had cutaneous edema localized to the left side of his face and an indurated swelling was more extensive in the periorbital tissue and lips. A preseptal cellulitis was diagnosed and oral and topical antibiotic treatment was begun. However, facial and periorbital edema were increased and reached the opposite side of the face, neck and region of the sternocleidomastoid muscle. Cutaneous anthrax lesion just below the lower eyelid in the left eye was seen. Cutaneous anthrax was diagnosed and the appropriate intravenous antibiotic treatment and oral steroid treatment were begun. Facial and periorbital edema regressed on the 10th day of treatment, but the scar tissue remained. The scabs fell down at the second month. The delay in diagnosis or misdiagnosis in the patients with anthrax might lead to fatal consequences for humans. The cutaneous anthrax should be considered at differential diagnosis of preceptal cellulitis.
\end{abstract}

\section{Introduction}

Anthrax is a zoonotic infection caused by Bacillus anthracis and it rarely affects humans. It is still an important health problem in Africa, Central Asia, the Middle East and Mediterranean countries [1]. In our country, it is seen in the East and Southeast Anatolia where uncontrolled breeding is most common region [3-6]. Currently, it has also been used as a biological warfare agent used in bioterrorist activities.

Anthrax is transmitted to humans via direct contact with infected animals or their tissues and their products, such as hides or wool, and inhalation of spores [1,2]. Anthrax is also considered as an occupational disease in especially underdeveloped countries because the persons dealing with livestock such as farmers, butchers and veterinarians are at greater risk of direct contact with infected tissues or animals. The most common form of anthrax is cutaneous anthrax, characterized by generalized severe edema of the involved site, and periocular involvement is rare [1-4]. Here, we report a case of cutaneous anthrax involving periorbital region misdiagnosed as preseptal cellulitis at the presentation.

\section{Case report}

A 14-year-old male patient was referred to emergency service of our university hospital with preliminary diagnosis of angioedema. He had cutaneous edema localized to the left side of his face. The indurated edema was more extensive in the periorbital region and lips. On initial examination, his corrected visual acuity was 20/20 in the both eyes. The intraocular pressure was $16 \mathrm{mmHg}$ in both eyes. Direct and indirect light reflexes were intact, without a relative afferent pupillary defect. The examinations of anterior segment and fundus and extraocular movements in both eye revealed no any pathological sign. A preseptal cellulitis was initially diagnosed based on the clinical and laboratory findings, and oral (ornidazole and ciprofloxacin) and topical antibiotic treatments (moxifloxacin drop and polymyxin B ointment) were beginned. At follow-up visits, edema at face and periorbital region was increased and involved both upper and lower eyelids and both side of face, neck and sternocleidomastoid region. $\mathrm{He}$ had fever on the first presentation and the fever increased to $38.8^{\circ} \mathrm{C}$. A small cutaneous black lesion just below the lower eyelid in the left eye was detected following careful observation. Based on the failure to respond to antibiotic treatment, information concerning the animal contact in medical story of the patient and the presence of specific black cutaneous lesion, a cutaneous anthrax was preliminary diagnosed by Department of Clinical Microbiology and Infectious Diseases in our hospital. The medical treatment was changed as intravenous penicillin G 250,000 IU/kg/day and chloramphenicol $100 \mathrm{mg} / \mathrm{kg} /$ day and oral steroid treatment of $1 \mathrm{mg} / \mathrm{kg} /$ day. The fact of the transformation of skin lesions to classical necrotic black lesions or scars, a dramatically response to the antibiotic treatment for 14 days and the confirmation of the presence of Bacillus anthracis, gram-positive bacillus with culture tests on smear taken from black crusted lesion provided the correct diagnosis for cutaneous anthrax. In systemic examination, no another form of anthrax was detected. Periorbital and facial edema regressed on the $10^{\text {th }}$ day of treatment, but the scar tissue remained. The scabs fell down at the second month. An informed consent was taken from the patient for presentation. The further follow-up visits were not possible because of patient inconsistency.

Correspondence to: Burak Turgut, MD, Associate Professor in Ophthalmology, Frrat University, School of Medicine, Department of Ophthalmology 23119, Elâziğ, Turkey; Tel: +904242333555; Fax: +90424; E-mail: drburakturgut@gmail.com

Key words: cutaneous anthrax, preseptal cellulitis, periorbital, oculocutaneous

Received: February 02, 2016; Accepted: March 04, 2016; Published: March 09, 2016 


\section{Conclusion}

Anthrax is a rare zoonotic infection caused by Bacillus anthracis. It is transmitted by the digestion of the meat of infected animals or the direct contact of the infected products such as skin and hair of the animal. The majority of the patients with anthrax are given a history of close contact with the animals [1-4]. In our country, the main transmission route is direct contact [4-6].

Humans might rarely be infected via the flies feeding on dead animals contaminated with bacterial spores. Anthrax has three clinical forms including cutaneous, inhalation, and gastrointestinal. The most common form, cutaneous anthrax $(95 \%)$ has a better prognosis. The mortality rate is around $20 \%$ without antibiotic treatment and $1 \%$ with antibiotic treatment. Although the frequency of anthrax decreases with various protective precautions, it is still an important health problem in Africa, Central Asia, the Middle East and Mediterranean countries [1]. In our country, it is seen in the East and Southeast Anatolia where uncontrolled breeding is most common region. Currently, it has been also used as a biological warfare agent used in bioterrorist activities [1-6].

Cutaneous anthrax often seems contact regions such as the head, face, neck and forearm. Cutaneous anthrax of periorbital region starts from the upper eyelid, reaches the lower eyelid and cheek, includes a serosanguinous exudation and improves with scab formation causing tissue retraction [7-9]. The first clinical sign of periorbital anthrax is a serious and generalized edema accompanied by induration in the infected region. Unlike orbital cellulitis, there is no pain, tenderness, and suppuration, indentations (pitting) or demarcation line in skin [49]. However, redness in the skin and hyperemia in conjunctiva may occur. Edema may spread to the face, neck and anterior wall of the chest, rarely to the trachea and it may cause respiratory distress by compression. In some cases with cutaneous anthrax, at the beginning, small papule, then vesicle within $24-48$ hours. Typically black anthrax ulcer develops within 1-2 weeks [2,4-9].

In our country, the most common localizations of cutaneous anthrax are the arm and finger, and its incidence at face and neck was reported as 20\% [3-6]. Especially, in the lesions of the face and neck, severe neck edema may develop in the second or third days. Also, lymphadenopathy may occur in the lymph nodes that drain these areas [1-4]. Fatality rate due to cutaneous anthrax without treatment is $20 \%$. The antibiotics to be used in the treatment of disease are preferably penicillin, and ciprofloxacin, tetracycline, amoxicillin and chloramphenicol. The treatment should be continued for 7-14 days, even 60 days for complicated cases. Systemically steroid treatment with prophylactic antibiotic may be used for severe periorbital edema. It has been reported that topical antibiotic therapy is not useful. As necrotic ulcer developed by toxins, the antibiotics used do not affect the lesion. However, the use of antibiotic prevents fatality. The excision of skin lesion should be avoided because excision may cause to increase the dimension of the lesion and systemic symptoms [1-6].

In the differential diagnosis of cutaneous anthrax, skin infections such as carbuncles, erysipelas, necrotizing cellulitis due to streptococcus and staphylococcus, tularemia, cutaneous tuberculosis, Leishmaniasis, cat scratch disease, primary syphilis chancre, necrotic herpes simplex infection and tropical ulcers should be considered [1-3].

Anthrax can be diagnosed by detecting bacillus with gram staining of a smear taken from the lesions and via culture growth and based on clinical and laboratory findings. Misdiagnosis or delay in diagnosis and therapy can cause visual loss, and fatality [2,7-9]. Each case
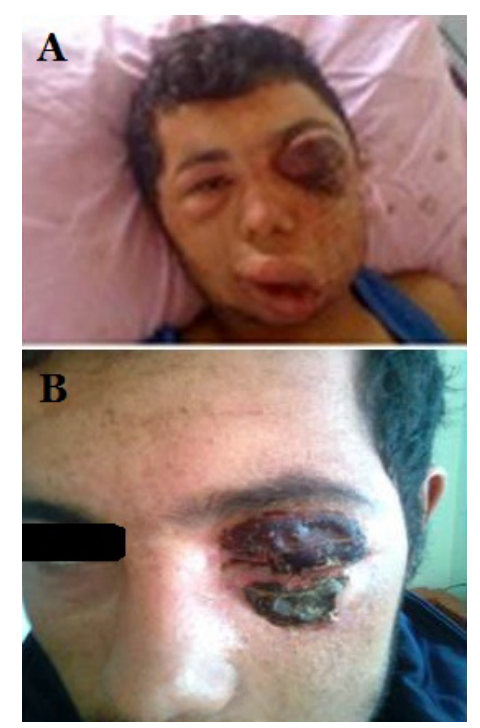

Figure 1. The post-treatment views of the patient at the first day (A) and the 1oth day (B) of the treatment.

diagnosed as preseptal cellulitis should be evaluated for periorbital cutaneous anthrax. If, in the patients presented as preseptal cellulitis, a painless necrotizing ulcer or a black eschar accompanied by periorbital or facial edema develops, cutaneous periorbital anthrax should be suspected. So, early diagnosis and treatment can prevent possible serious and severe ocular complications such as cicatricial ectropion and keratopathy, and systemical complications such as septicaemia and meningitis. We report this case because ophthalmologists and physicians might recognize and aware of the signs and symptoms of cutaneous periorbital anthrax (Figure 1).

\section{Authorship and contribution}

All authors listed on the title page made significant contributions to this manuscript.

\section{Acknowledgements}

None

\section{Funding}

No funding was received for this study.

\section{Competing interest}

The authors have no conflict of interest or financial relationships related to this manuscript.

\section{References}

1. Hart CA, Beeching NJ (2002) A spotlight on anthrax. Clinics in Dermatology 20: 365 375. [Crossref]

2. Lew DL (2000) Bacillus anthracis (Anthrax). In: Mandell GL, Bennett JE, Dolin R, eds. Principles and Practice of Infectious Diseases. New-York: Churchill Livingstone Inc. 2215-2220.

3. Carucci JA, McGovern TW, Norton SA, Daniel CR, Elewski BE, et al. (2002) Cutaneous anthrax management algorithm. J Am Acad Dermatol 47: 766-769. [Crossref]

4. Irmak H, Buzgan T, Karahocagil MK, Sakarya N, Akdeniz H, et al. (2003) Cutaneus manifestations of Anthrax in Eastern Anatolia: a review of 39 cases. Acta Med Okayama 57: 235-240. [Crossref]

5. Demirdag K, Ozden M, Saral Y, Kalkan A, Kilic SS, et al. (2003) Cutaneous Anthrax 
Gül FC (2016) Preseptal cellulitis as a rare presentation of the oculocutaneous anthrax

in adults: a review of 25 cases in the Eastern Anatolian Region of Turkey. Infection 31: 327-330. [Crossref]

6. Oncül O, Ozsoy MF, Gul HC, Koçak N, Cavuslu S, et al. (2002) Cutaneous anthrax in Turkey: a review of 32 cases. Scand J Infect Dis 34: 413-416. [Crossref]

7. Celebi S, Celebi H, Celiker UO, Kandemir B, Alagöz G, et al. (1997) Anthrax as the cause of preseptal cellulitis. Acta Ophthalmol Scand 75: 462-463. [Crossref]
8. Caça I, Cakmak SS, Unlü K, Sakalar YB, KadiroGlu AK (2004) Cutaneous anthrax on eyelids. Jpn J Ophthalmol 48: 268-271. [Crossref]

9. Tekin R, Ari S, Dal T, Kaya S, Kortak MZ, et al. (2013) Evaluation of cutaneous palpebral anthrax. Cutan Ocul Toxicol 32: 294-298. [Crossref]

Copyright: (C2016 Gül FC. This is an open-access article distributed under the terms of the Creative Commons Attribution License, which permits unrestricted use, distribution, and reproduction in any medium, provided the original author and source are credited. 